\title{
Interactive, multi-modality image registrations for combined MRI/MRSI-planned HDR prostate brachytherapy
}

\author{
Galen Reed', J. Adam Cunha, PhD², Susan Noworolski, PhD', John Kurhanewicz, PhD', Daniel Vigneron, PhD', \\ I-Chow Hsu, MD², Jean Pouliot, PhD² \\ IDepartment of Radiology and Biomedical Imaging, 2Department of Radiation Oncology, University of California, San Francisco, USA
}

\begin{abstract}
Purpose: This study presents the steps and criteria involved in the series of image registrations used clinically during the planning and dose delivery of focal high dose-rate (HDR) brachytherapy of the prostate.

Material and methods: Three imaging modalities - Magnetic Resonance Imaging (MRI), Magnetic Resonance Spectroscopic Imaging (MRSI), and Computed Tomography (CT) - were used at different steps during the process. MRSI is used for identification of dominant intraprosatic lesions (DIL). A series of rigid and nonrigid transformations were applied to the data to correct for endorectal-coil-induced deformations and for alignment with the planning CT. Mutual information was calculated as a morphing metric. An inverse planning optimization algorithm was applied to boost dose to the DIL while providing protection to the urethra, penile bulb, rectum, and bladder. Six prostate cancer patients were treated using this protocol.

Results: The morphing algorithm successfully modeled the probe-induced prostatic distortion. Mutual information calculated between the morphed images and images acquired without the endorectal probe showed a significant $(p=0.0071)$ increase to that calculated between the unmorphed images and images acquired without the endorectal probe. Both mutual information and visual inspection serve as effective diagnostics of image morphing. The entire procedure adds less than thirty minutes to the treatment planning.

Conclusion: This work demonstrates the utility of image transformations and registrations to HDR brachytherapy of prostate cancer.
\end{abstract}

Key words: MRSI, HDR, prostate, image registration.

\section{Purpose}

Studies have shown that greater control of localized prostatic tumors is directly correlated with radiation doses delivered [1-3]. However, excessive dose to the normal tissues causes side effects [4-6]. High dose rate (HDR) brachytherapy can provide a focal dose escalation, and prior studies have shown that combined external beam radiation therapy (EBRT) and HDR brachytherapy yields favorable outcomes, particularly in patients with locally advanced disease [7-9]. We have recently initiated an HDR brachytherapy clinical protocol in which a combination of magnetic resonance imaging (MRI) and magnetic resonance spectroscopic imaging (MRSI) is used to define dominant intraprostatic lesions (DIL). This information is used to perform dose escalation of the DIL without compromising the dose coverage of the prostate nor the protection to the urethra, rectum, and bladder. There are two difficulties, however, inherent in this approach. The first is the merging of information from the staging MRI/MRSI exam with the anatomic computed tomography (CT) exam required for treatment planning; the second is the capacity of the treatment modality/treatment planning system to deliver radiation doses in a precise fashion.

The combined MRI/MRSI exam has been shown to be an effective method of detecting and localizing intraprostatic lesions [10-13]. The high sensitivity and specificity yielded by this exam has demonstrated great utility in focal treatment via ${ }^{125} \mathrm{I}$ brachytherapy implants [14] and combined HDR brachytherapy + EBRT $[15,16]$.

However, since the endorectal probe used for MRI/ MRSI acquisition can induce significant prostatic compression and deformation, a non-rigid transformation is required to accurately register the probe-in MRSI data with the probe-out CT images used for treatment planning. To model this deformation, prior studies have implemented finite-element based biomechanical simulations [17,18], elastic spline deformation [19], thin plate spline deformation [19-23], symmetric forces algorithms [24], Newton-
Address for correspondence: Galen Reed, Department of Radiology and Biomedical Imaging, University of California, San Francisco, California 94107, USA, Phone: 001 (415) 514-4802,

e-mail: galen.reed@ucsf.edu
Received: 18.01 .11

Accepted: 11.03 .11

Published: 31.03 .11 
Ralphson algorithms [25], and in-plane linear scaling assuming constant volume [14]. Kim et al. [26] showed that the deformation induced by the inflatable endorectal coil is negligible in the $\mathrm{z}$ (superior/inferior) dimension since the induced distortions are less than the axial MRI slice thickness of $3 \mathrm{~mm}$. The same study also showed that the coil induces a non-negligible rotation about the $x$ (right/ left) axis. These findings suggest that an $x$ axis rotation followed by an axial in-plane non-rigid deformation sufficiently models the prostatic deformation induced by the endorectal coil.

In this study, we developed and implemented a new method combining rigid translations (for centroid alignment), rotations (to model the $x$ axis rotation), and an inplane non-rigid control-point based morphing method that utilizes a local weighted-mean transformation [27]. Additionally, we present the steps and criteria involved in the series of image registrations used clinically during the planning of the dose delivery process.

\section{Material and methods}

For each patient enrolled in the study, a pre-therapy staging MRI/MRSI exam using an endorectal probe (i.e. probe in, or PI) was acquired. The MRI and MRSI images were registered so that the MRSI-defined DIL could be delineated on the anatomic MRI volume. An axial MRI series with the endorectal probe not inserted (i.e. probe out, or MRI-PO) was also acquired. Pairs of points at corresponding anatomic features on the MRI-PI and MRI-PO series were chosen and used to determine the PI-PO transformation. This transformation algorithm was used to correlate spectroscopic data (MRSI-PI) to the MRI-PO data. Mutual information was calculated to measure warping accuracy. On the HDR brachytherapy treatment day, the planning CT and the MRSI-PO were imported into the brachytherapy planning system and registered to each other. The prostate anatomy alone was used to guide the fusion. The target and organs at risk were delineated using the CT, while the DIL was contoured using spectroscopic information from the MRSI-PO. Catheters were then digitized, and the plan was optimized using the Inverse Planning by Simulated Annealing (IPSA) algorithm [16]. The optimization was instructed to provide a clinically effective dose to the entire prostate while concurrently delivering a dose escalation to the DILs. This was done without increasing the dose to surrounding non-cancerous tissues. Once the plan was approved, the dose for the first fraction was delivered.

\section{Subjects}

Six patients were scanned and subsequently treated with this protocol. Their age ranged from 44 to 80 years old (mean 61). All patients had biopsy-proven Gleason $3+3$ disease, and five of the six subjects showed Gleason $3+4$ pattern in histological analysis. The mean PSA measurement for all subjects was $8.8 \mathrm{ng} / \mathrm{mL}$ (ranging from $4.3 \mathrm{ng} / \mathrm{mL}$ to $19.5 \mathrm{ng} / \mathrm{mL}$ ). Written informed consent was obtained for all subjects following a protocol approved by the UCSF Committee of Human Research.

\section{Identification of DIL with MRS and MRI}

MR imaging was acquired with a GE 3T SIGNA scanner (GE Medical Systems, Waukesha, WI) using body-coil excitation. The GE 8-channel pelvic phased array and Medrad endorectal coil (Medrad, Pittsburgh, PA) were used for signal reception. Axial $\mathrm{T}_{1}$-weighted images were acquired with TR/TE $=600 / 12$. Fast spin echo $T_{2}$-weighted images were acquired $(\mathrm{TR} / \mathrm{TE}=6000 / 102,14 \mathrm{~cm}$ FOV, $256 \times 256$ matrix) in the axial, sagittal, and coronal planes. Images and spectroscopic data were first acquired with the endorectal probe inserted. The probe was then removed, and an additional sagittal locator and an axial FSE $\mathrm{T}_{2}$ image data set were then acquired using the pelvic phased array for signal reception.

Spectroscopic data was acquired using a specialized prostate 3D MRSI sequence [28,29] with a $12 \times 8 \times 8$ acquisition grid, $5.4 \mathrm{~mm}$ isotropic resolution yielding $0.157 \mathrm{~cm}^{3}$ voxels. Water and lipid suppression was achieved using dual-band spectral spatial pulses [30]. Very-selective spatial saturation (VSS) pulses were used to suppress periprostatic lipids [31]. In each voxel 1024 points were acquired over a $1000 \mathrm{~Hz}$ frequency domain. The k-space data was zerofilled in the superior/inferior and anterior/posterior dimensions to a final array size of $12 \times 16 \times 16$. The spectral data was then apodized with a $3 \mathrm{~Hz}$ Gaussian filter, Fourier transformed, baseline corrected, frequency aligned, and peaks numerically-integrated. Numerical integration of each of the prostate metabolite peaks (choline, creatine, and citrate) and the suppressed water peak was performed using the known frequency positions of each of these peaks [32].

Spectroscopic voxels were classified using the standardized scoring system proposed by Jung et al. [33] where 1 = definitely normal, 2 = probably normal, 3 = equivocal, $4=$ probably abnormal, and $5=$ definitely abnormal. After the generation of these scores by a trained reader, grayscale images of these scores were created at the MRSI resolution, so that the suspicious voxels could be delineated by color on the high-resolution $\mathrm{T}_{2}$ volume (Fig. 1). All peripheral zone voxels were tagged; central zone voxels were only tagged when suspicious for malignancy.

\section{Warping the MRSI grid}

\section{Rotation}

Sagittal images acquired with and without the endorectal probe were analyzed to determine the probe-induced rotation of the prostate about the $x$ axis. The prostatic alignment of each series is determined by the margin of the peripheral zone and central zone as shown in Fig. 2. The image volumes are rotated by the net rotation angle $\theta_{\text {probein }}-\theta_{\text {probeout }}$ using tri-linear interpolation.

\section{Translation}

Prostatic margins were then hand-drawn on both the axial probe-out volume and the rotated axial probe-in volume. Masks were drawn on the ten to twelve corresponding slices in each volume. The centroids of the masks were calculated, and the rotated axial probe-in volumes were translated so their centroids line up with the centroid of 

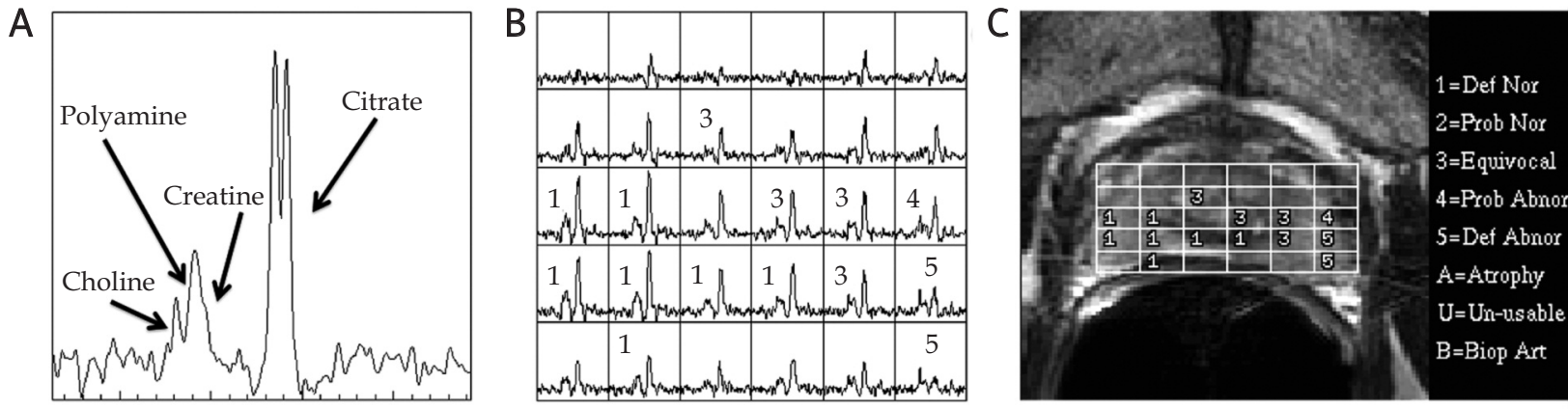

Fig. 1. A shows a typical healthy prostate NMR spectrum, with high citrate and polyamine sigal and low choline. B shows the spectral data of a patient enrolled in the study; elevated choline and reduced citrate in the left peripheral zone are indicative of malignancy. $C$ shows the suspicious regions (scores of 3,4 , and 5 ) delineated on the $T_{2}$ image. All peripheral zone voxels were tagged; central zone voxels were only tagged when suspicious for malignancy. Only intraprostatic voxels are shown
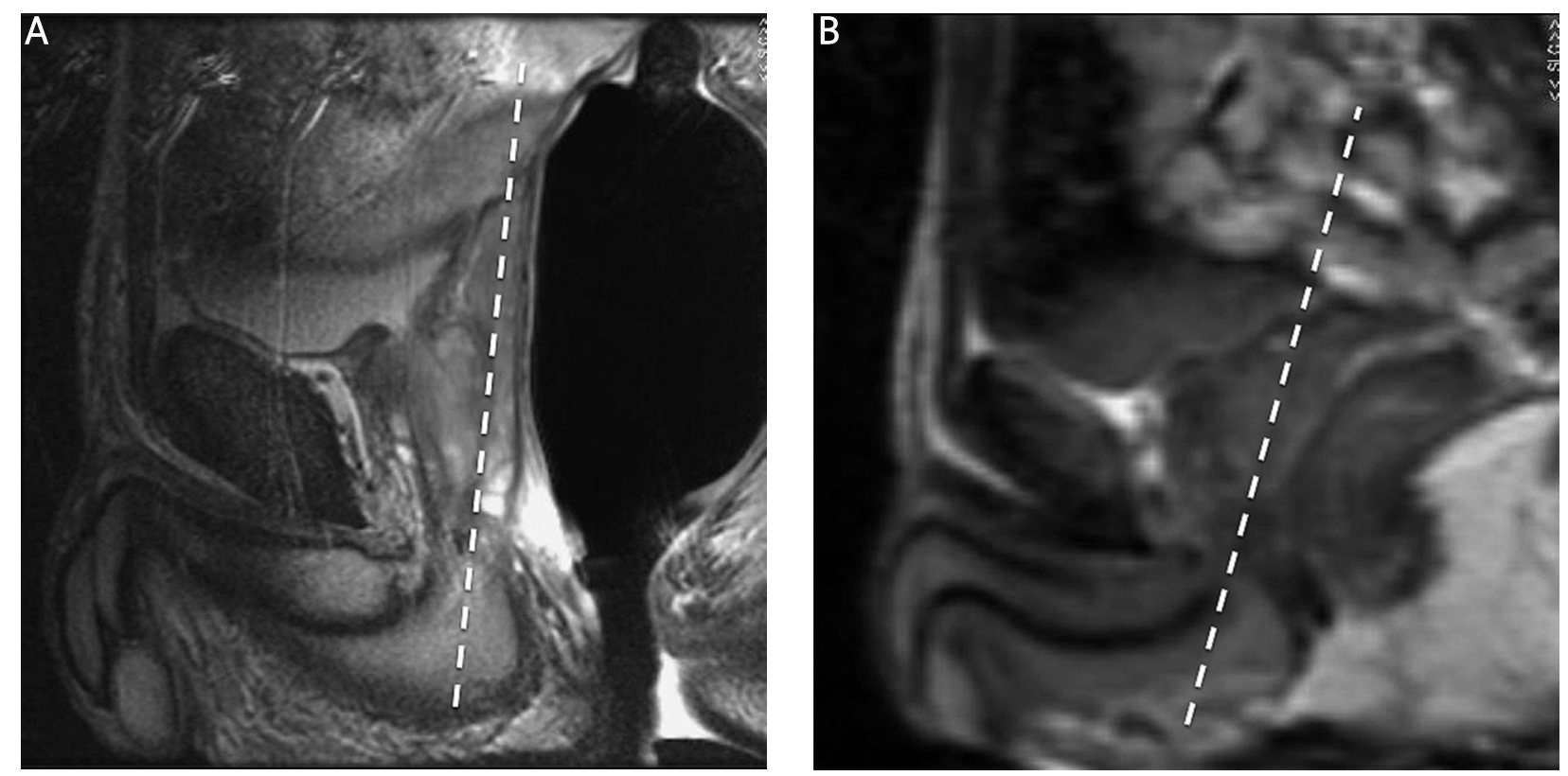

Fig. 2. Mid-sagittal probe-in (A) and probe-out (B) images showing the prostatic rotation induced by the endorectal coil. The angles were measured between the y axis and the peripheral zone/central zone margin denoted by the dotted line

the probe-out volume. Defining $\mathrm{R}_{\mathrm{CI}}$ and $\mathrm{R}_{\mathrm{CO}}$ as the centers and $C_{\mathrm{I}}$ and $\mathrm{C}_{\mathrm{O}}$ as the prostate centroids of the probe-in and probe-out volumes respectively, the translation

$$
\Delta R=R_{C I}-R_{C O}+C_{O}-C_{I}
$$

aligns the prostate in both volumes provided they are acquired using the same landmark.

\section{Non-rigid deformation}

After the rotation and translations were applied, a nonrigid deformation was used to model the prostatic deformation induced by the endorectal coil. To model this deformation, the probe-in and probe-out $\mathrm{T}_{2}$ imaging series were compared slice-wise. In each slice, control point pairs were assigned to matching anatomical landmarks within the prostate (Fig. 3). The control point locations were assigned using the MATLAB control point selection tool. These point pairs were then used to define a local weighted-mean [27] PI-PO transformation. This non-rigid transformation placed higher weighting on image regions with a higher control point density. Therefore, the operator could apply more control points onto the regions where higher accuracy was required with the registration. Examples are regions of higher prostatic compression (the posterior aspect) and regions of suspected malignancy. The transformation was applied to the MRI-PI volume (to calculate morphing accuracy) and to the MRSI-PI data. Finally, the MRSI-PI data (after rotation, translation, and deformation) were delineated on the probe-out $\mathrm{T}_{2}$ volume (Fig. 4).

\section{Evaluation}

Mutual information (MI) was calculated between the MRI-PO images and both the warped and non-warped MRI-PI images after rotations and translations were applied. This value was calculated similar to previous 

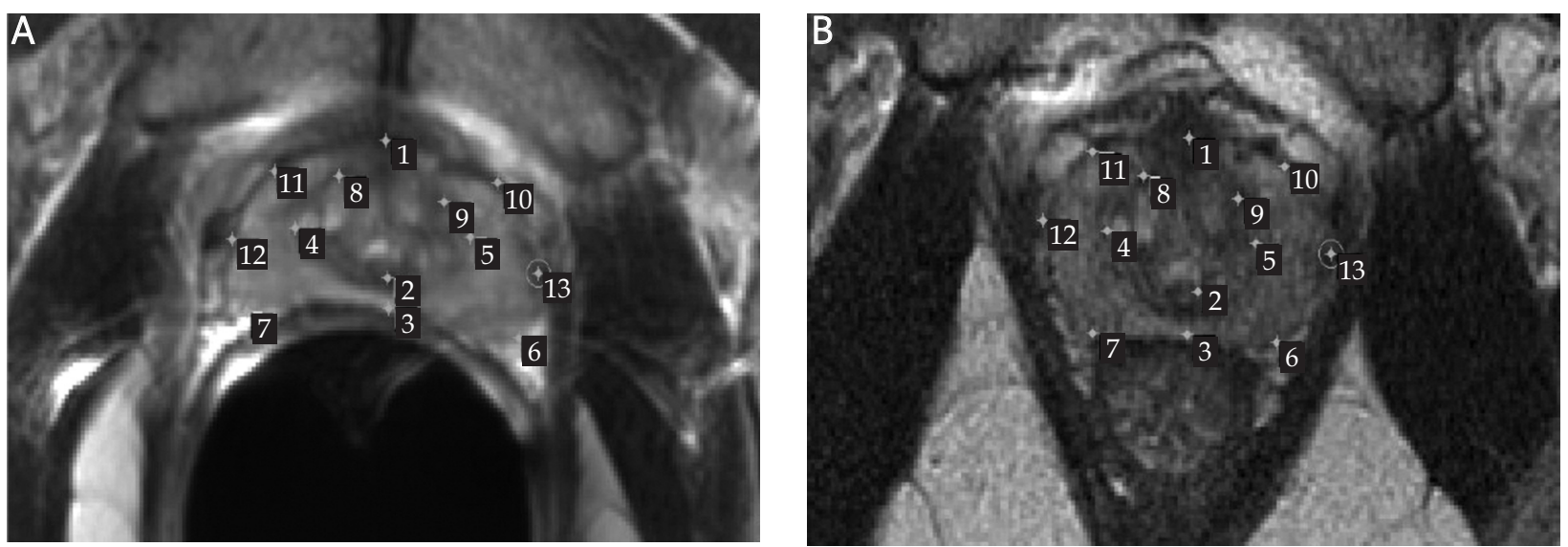

Fig. 3. Probe-in (A) and probe-out (B) axial slices showing control point placement. The probe-in volume is rotated by the prostatic angle so the slices are assumed to be coplanar. Despite the lower signal-to-noise ratio on the probe-out image, numerous landmarks are still identifiable on both images

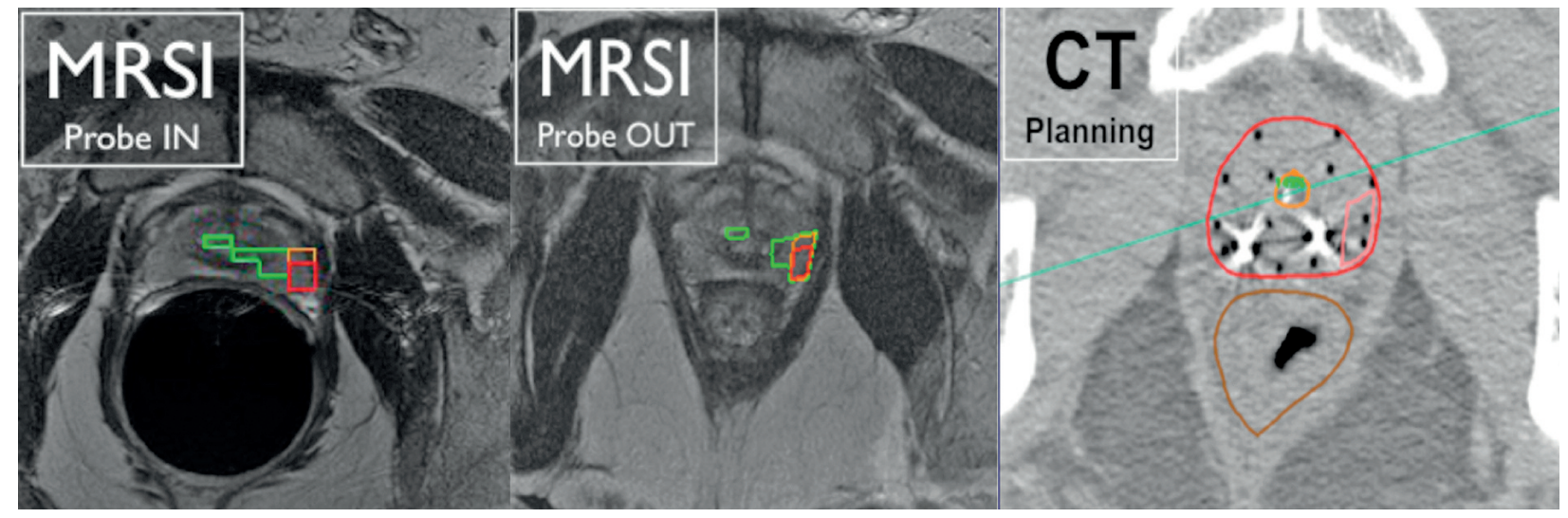

Fig. 4. The left image shows the original acquisition image with the suspicious regions delineated. The center image shows the axial probe-out volume with the rotate, translated, and warped spectral delineations. MRSI scores of 4 and 5 are delineated on the planning CT (right)

works [34] using the histogram method. MI was calculated only in the smallest-fitting rectangular boundary around the hand-drawn mask for each slice so that the erroneous morphing in the extra-prostatic regions (where no control points are placed) did not excessively sway the metric.

\section{Target delineation for dose planning optimization}

\section{MRSI - planning CT registration}

On the HDR brachytherapy treatment day, a CT scan with $3 \mathrm{~mm}$ slice thickness was acquired immediately after the patient recovered from the implant procedure. The imaging volume included the whole of the prostate and was limited superiorly to include the bladder and inferiorly to visualize all the catheters down to the perineum. The planning CT and the MRSI-PO were imported in the brachytherapy planning system (OncentraBrachy ${ }^{\mathrm{TM}}$, Nucletron, Veenendal, the Netherlands), and a rigid body registration was obtained. The prostate anatomy alone was used to guide the fusion, since the prostatic position can vary with respect to extra-prostatic landmarks. Typically, 3 pairpoints were defined: two by the urethra in the base and apex areas, and a third one more lateral to the prostate in the median plane. Target and organs at risk were delineated on the CT while the DILs were contoured on the MRSI-PO using spectroscopic information. Catheters were then digitized, the plan was optimized using IPSA, and the dose for the first fraction was delivered.

\section{Definition of volumes of interest}

Clinical target volumes (prostate) and organs at risk (urethra, bladder, rectum and bulb) were contoured on each CT slice (Fig. 5A). The DILs - defined by MRSI voxel scores of 4 or 5 - were manually contoured on the CT, but the transparency level was adjusted to make the MRS information visible on the CT (Fig. 4 right). After the catheters were digitized on the $\mathrm{CT}$, all information requested for the optimization of the dose distribution was available (Fig. 5B).

\section{Dose optimization with inverse planning IPSA}

The inverse planning optimization algorithm was then used to increase the dose delivered to the dominant intra- 


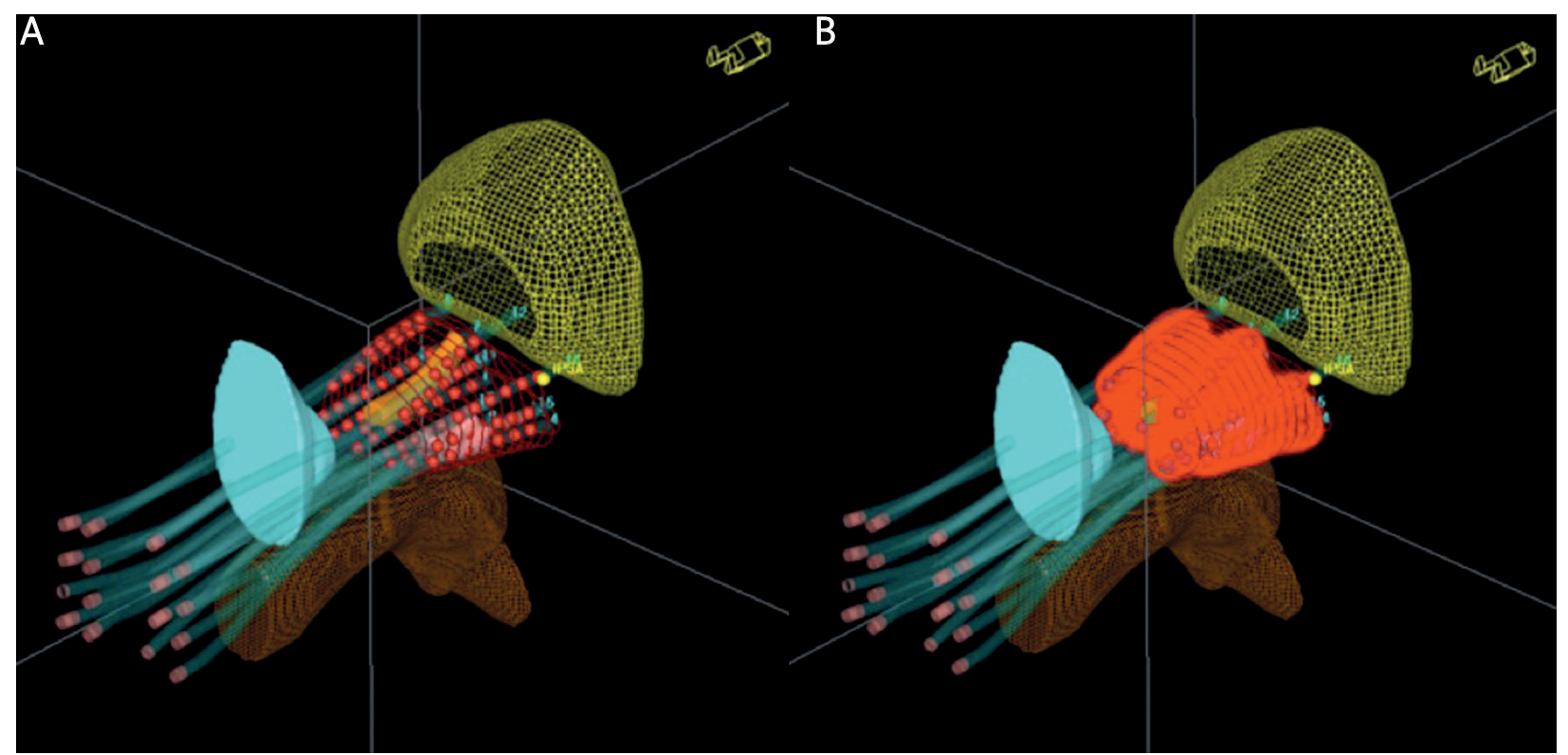

Fig. 5. Digitized catheters and delineated target organs: rectum (brown), urinary bladder (yellow mesh), and urethra (solid yellow). The MRSI-defined DIL is shown in pink on the left, and the right figure shows an isodose contour

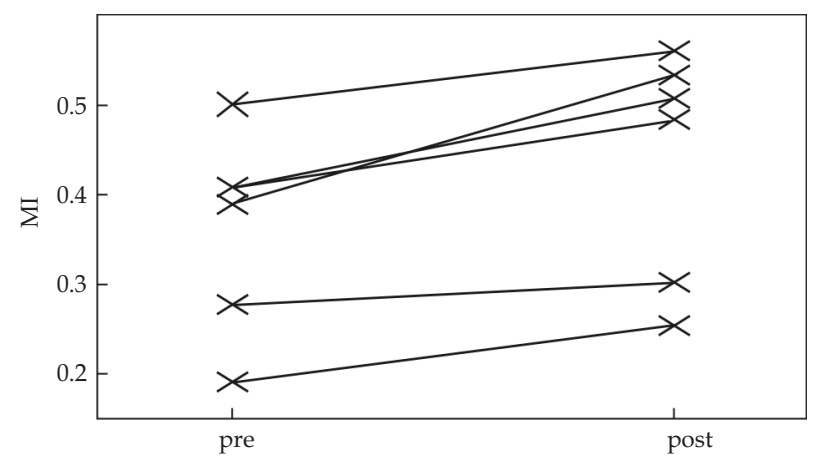

Fig. 6. Mutual information before and after applying the described transformations to the MRI volumes for each of the six patients in the trial. Each data point represents the mean values of all slices in the prostate

prostatic lesions defined by MRI/MRSI while providing the usual dose coverage of the prostate and the protection to the urethra, rectum, bulb and bladder.

A class solution - a set of weights provided to the optimization to guide it to satisfy the dose requirements of each region of interest - was previously developed [15] for dose escalation of a DIL defined by combined MRI/MRSI in inverse planned HDR prostate brachytherapy. Using this class solution, a certain level of DIL-boost was feasible for the majority of patients under the RTOG-0321 dosimetric requirements depending on rectal and bladder doses. The class solution in inverse planned HDR prostate brachytherapy for dose escalation of a DIL defined by combined MRI/MRSI was an excellent starting point to explore a customized set of dose constraints to obtain a satisfactory treatment plan for each patient in the ongoing protocol.

\section{Results and Discussion}

The complete procedure was clinically integrated and has been used for the treatment of six HDR brachytherapy patients with MRSI information. Out of the 6 patients scanned, the mean prostatic rotation induced by the coil was $20 \pm 9$, and the mean gland volume was $27+14 \mathrm{~cm}^{3}$. Urethra, peripheral zone margins, prostate boundaries, and various hyper/hypointense features on the images served as effective landmarks for the MRI-PI-MRI-PO fusion (Fig. 3). At least 12 point pairs - selected primarily from regions with high deformation, such as the posterior aspect of the peripheral zone, and regions of spectroscopic abnormality - are required as inputs to the MATLAB image transformation function. Visual inspection as well as the computed MI (Fig. 6) served as effective diagnostics of the morphing. MI showed $25 \% \pm 13 \%$ increase for the six patients. A paired $t$-test showed this improvement to be significant $(p=0.0071)$. On the day of the first fraction, performing the MRSI-CT fusion was followed by the delineation of the DIL adding less than fifteen minutes to the entire planning process. The 3 pair-points registration procedure is considered valid when the sum of squared distances between each pair-point is less than $2 \mathrm{~mm}$. A careful visual inspection of the fusion in the prostate area was also performed.

\section{Conclusions}

An MRSI to MRI/CT alignment protocol was developed to exploit the high specificity of combined MRI/MRSI for detecting and localizing prostate cancer within the prostate, and to accurately transfer this information to the planning images. This makes information about the prostate cancer location routinely available, and allowing the use of inverse 
planning IPSA to boost dominant intraprostatic lesions during HDR brachytherapy, while preserving the prostate coverage and keeping the dose delivered to the organs at risk at the same level compared to an inverse planned dose distribution without DIL boost. Information from one image data set could be accurately transferred to another in the series of MRS $\rightarrow$ MRI $\rightarrow$ CT. This workflow was routinely used for the dose planning, including DIL boost. This work illustrates the clinical benefit of image registration tools.

\section{Acknowledgements}

This work was supported by the Department of Defense Grant, IDPCRP-DAMD17-PC02 (PC030909)), and National Institute of Health Grants R01 CA79980, R01 CA59897 and R01 CA11129.

\section{References}

1. Hanks GE, Hanlon AL, Schultheiss TE et al. Dose escalation with 3D conformal treatment: five year outcomes, treatment optimization, and future directions. Int J Radiat Oncol Biol Phys 1998; 41: 501-510.

2. Perez CA, Hanks GE, Leibel SA et al. Localized carcinoma of the prostate (Stages T1B, T1C, T2, and T3). Review of management with external beam radiation therapy. Cancer 1993; 72: 3156-3173.

3. Hanks GE. Optimizing the radiation treatment and outcome of prostate cancer. Int J Radiat Oncol Biol Phys 1985; 11: 12351245 .

4. Gellekom MPV, Moerland MA, Vulpen MV et al. Quality of life of patients after permanent prostate brachytherapy in relation to dosimetry. Int J Radiat Oncol Biol Phys 2005; 63: 772-780.

5. Kang SK, Chou RH, Dodge RK et al. Acute urinary toxicity following transperineal prostate brachytherapy using a modified Quimby loading method. Int J Radiat Oncol Biol Phys 2001; 50: 937-945.

6. Yoshioka Y, Konishi K, Oh RJ et al. High-dose-rate brachytherapy without external beam irradiation for locally advanced prostate cancer. Radiother Oncol 2006; 80: 62-68.

7. Galalae RM, Kovács G, Schultze J et al. Long-term outcome after elective irradiation of the pelvic lymphatics and local dose escalation using high-dose-rate brachytherapy for locally advanced prostate cancer. Int J Radiat Oncol Biol Phys 2002; 52: 81-90.

8. Martinez AA, Gustafson G, Gonzalez J et al. Dose escalation using conformal high-dose-rate brachytherapy improves outcome in unfavorable prostate cancer. Int J Radiat Oncol Biol Phys 2002; 53: 316-327.

9. Martinez A, Gonzalez J, Spencer W et al. Conformal high dose rate brachytherapy improves biochemical control and cause specific survival in patients with prostate cancer and poor prognostic factors. J Urology 2003; 169: 974-980.

10. Kurhanewicz J, Vigneron D, Hricak H et al. Three-dimensional H-1 MR spectroscopic imaging of the in situ human prostate with high (0.24-0.7-cm3) spatial resolution. Radiology 1996; 198 : 795-805.

11. Scheidler J, Hricak H, Vigneron DB et al. Prostate cancer: localization with three-dimensional proton MR spectroscopic imaging-clinicopathologic study. Radiology 1999; 213: 473-480.

12. Kurhanewicz J, Vigneron DB, Nelson SJ. Three-dimensional magnetic resonance spectroscopic imaging of brain and prostate cancer. Neoplasia 2000; 2: 166-189.

13. Coakley FV, Qayyum A, Kurhanewicz J. Magnetic resonance imaging and spectroscopic imaging of prostate cancer. J Urology 2003; 170 (Suppl 1): S69-S76.
14. Zaider M, Zelefsky MJ, Lee EK et al. Treatment planning for prostate implants using magnetic-resonance spectroscopy imaging. Int J Radiat Oncol Biol Phys 2000; 47: 1085-1096.

15. Kim Y, Hsu ICJ, Lessard E et al. Class solution in inverse planned HDR prostate brachytherapy for dose escalation of DIL defined by combined MRI/MRSI. Radiother Oncol 2008; 88: 148-155.

16. Pouliot J, Kim Y, Lessard E et al. Inverse planning for HDR prostate brachytherapy used to boost dominant intraprostatic lesions defined by magnetic resonance spectroscopy imaging. Int J Radiat Oncol Biol Phys 2004; 59: 1196-1207.

17. Alterovitz R, Goldberg K, Pouliot J et al. Registration of MR prostate images with biomechanical modeling and nonlinear parameter estimation. Med Phys 2006; 33: 446-454.

18. Crouch J, Pizer S, Chaney E et al. Automated finite-element analysis for deformable registration of prostate images. IEEE T Med Imaging 2007; 26: 1379-1390.

19. Kaus MR, Brock KK, Pekar V et al. Assessment of a modelbased deformable image registration approach for radiation therapy planning. Int J Radiat Oncol Biol Phys 2007; 68: 572-580.

20. Chappelow J, Both S, Viswanath $S$ et al. Computer-assisted targeted therapy (CATT) for prostate radiotherapy planning by fusion of CT and MRI. Medical Imaging: Visualization, ImageGuided Procedures, and Modeling 2010; 7625: 76252C.

21. Cheung MR, Krishnan K. Interactive Deformation Registration of Endorectal Prostate MRI Using ITK Thin Plate Splines. Acad Radiol 2009; 16: 351-357.

22. Venugopal N, McCurdy B, Hnatov A et al. A feasibility study to investigate the use of thin-plate splines to account for prostate deformation. Phys Med Biol 2005; 50: 2871-2885.

23. Lian J, Xing L, Hunjan S et al. Mapping of the prostate in endorectal coil-based MRI/MRSI and CT: a deformable registration and validation study. Med Phys 2004; 31: 3087-3094.

24. Godley A, Ahunbay E, Peng C et al. Automated registration of large deformations for adaptive radiation therapy of prostate cancer. Med Phys 2009; 36: 1433-1441.

25. Wu X, Dibiase SJ, Gullapalli R et al. Deformable image registration for the use of magnetic resonance spectroscopy in prostate treatment planning. Int J Radiat Oncol Biol Phys 2004; 58: 1577-1583.

26. Kim Y, Hsu ICJ, Pouliot J et al. Expandable and rigid endorectal coils for prostate MRI: Impact on prostate distortion and rigid image registration. Med Phys 2005; 32: 3569-3578.

27. Goshtasby A. Image registration by local approximation methods. Image Vision Comput 1988; 6: 255-261.

28. Cunningham C, Vigneron D, Marjanska M et al. Sequence design for magnetic resonance spectroscopic imaging of prostate cancer at 3 T. Magn Reson Med 2005; 53: 1033-1039.

29. Chen A, Cunningham C, Ozturk-Isik E et al. High-speed 3T MR spectroscopic imaging of prostate with flyback echo-planar encoding. Magn Reson Imaging 2007; 25: 1288-1292.

30. Schricker AA, Pauly JM, Kurhanewicz J et al. Dualband spectral-spatial RF pulses for prostate MR spectroscopic imaging. Magn Reson Med 2001; 46: 255-261.

31. Tran TKC, Vigneron DB, Sailasuta N et al. Very selective suppression pulses for clinical MRSI studies of brain and prostate cancer. Magn Reson Med 2000; 43: 23-33.

32. Nelson SJ, Brown TR. A new method for automatic quantification of 1-D Spectra with low signal to noise ratio. J Magn Reson 1987; 75: 229-243.

33. Jung JA, Coakley FV, Vigneron DB et al. Prostate depiction at endorectal MR spectroscopic imaging: investigation of a standardized evaluation system. Radiology 2004; 233: 701-708.

34. Maes F, Collignon A, Vandermeulen D et al. Multimodality image registration by maximization of mutual information. IEEE Trans Med Imaging 1997; 16: 187-198. 